\title{
Correlating tumor-infiltrating lymphocytes and lung cancer stem cells: a cross-sectional study
}

\author{
Valentina Masciale ${ }^{1 \#}$, Giulia Grisendi" ${ }^{2 \#}$, Federico Banchelli ${ }^{3}$, Roberto D'Amico ${ }^{3}$, Antonino Maiorana ${ }^{4}$, \\ Pamela Sighinolfi ${ }^{4}$, Massimo Pinelli ${ }^{5}$, Eleonora Lovati ${ }^{1}$, Alessandro Stefani ${ }^{1}$, Uliano Morandi ${ }^{1}$, Massimo \\ Dominici $^{2 *}$, Beatrice Aramini ${ }^{1 *}$
}

${ }^{1}$ Division of Thoracic Surgery, ${ }^{2}$ Division of Oncology, ${ }^{3}$ Center of Statistic, ${ }^{4}$ Institute of Pathology, ${ }^{5}$ Division of Plastic Surgery, Department of Medical and Surgical Sciences for Children \& Adults, University of Modena and Reggio Emilia, Modena, Italy

Contributions: (I) Conception and design: B Aramini, M Dominici; (II) Administrative support: B Aramini, U Morandi; (III) Provision of study materials or patients: B Aramini, V Masciale, U Morandi, A Stefani, M Pinelli, A Maiorana, P Sighinolfi; (IV) Collection and assembly of data: V Masciale, G Grisendi; (V) Data analysis and interpretation: F Banchelli, R D’Amico, B Aramini, V Masciale, G Grisendi, M Dominici; (VI) Manuscript writing: All authors; (VII) Final approval of manuscript: All authors.

\#These authors contributed equally to this work.

*These authors contributed equally for the last authorship.

Correspondence to: Beatrice Aramini, MD, PhD. Division of Thoracic Surgery, Department of Medical and Surgical Sciences for Children \& Adults, University of Modena and Reggio Emilia, Via Largo del Pozzo, 71, 41124 Modena, Italy. Email: beatrice.aramini@unimore.it.

Background: Lung cancer stem cells (LCSCs) are endowed with high aldehyde dehydrogenase (ALDH) expression and play roles in tumor proliferation, metastasis, and drug resistance. Their elusive nature may allow them to escape the immune response by tumor-infiltrating lymphocytes (TILs), which can positively affect the outcome in non-small cell lung cancer (NSCLC) patients. Despite independent investigations on both LCSCs and TILs, the relationship between the two has been very marginally considered. We analyzed whether these two cell types may be related as a prerequisite for novel diagnostic and therapeutic approaches. Methods: In this cross-sectional study, NSCLC human surgical specimens from 12 patients were tested by ALDEFLUOR assay to identify ALDH ${ }^{\text {high }}$ cells. Fluorescence-activated cell sorting (FACS) analyses for CD3+, CD4+, and CD8+ TILs were performed in combination with immunohistochemistry evaluation.

Results: Statistically positive correlations were found between ALDH+ and CD8+, and between ALDH+ and CD3+ cells populations; no correlation was found between $\mathrm{ALDH}+$ and $\mathrm{CD} 4+$ cells. The expression of CD3+ and CD8+ by cells accounted for $40.1 \%$ and $58.7 \%$, respectively, of the variability of ALDH+ cell expression by an R-squared index, which highlights the strong correlation between TILs and LCSCs. Immunohistochemistry revealed 6-25\% positive cells.

Conclusions: We report a correlation between cytotoxic TILs and LCSCs, which may contribute to the future development of targeted therapies focusing on the different roles of lymphocytes against lung cancer.

Keywords: Tumor-infiltrating lymphocytes (TILS), cancer stem cells (CSCs), cancer stem-like cells, non-small cell lung cancer (NSCLC), immunotherapy

Submitted Aug 05, 2019. Accepted for publication Oct 25, 2019.

doi: $10.21037 /$ atm.2019.11.27

View this article at: http://dx.doi.org/10.21037/atm.2019.11.27

\section{Introduction}

Chemotherapy agents generally contain but do not totally eliminate tumor cells in non-small cell lung cancer
(NSCLC) patients (1). This action is also related to a subpopulation of cells that can escape from such therapies and exhibit lung cancer stem cell (LCSC) characteristics, including self-renewal, multiple differentiation and tumor 
initiation (2). The identification of LCSCs has been the subject of several investigations to define their phenotypic and biological properties $(3,4)$. Aldehyde dehydrogenase (ALDH) activity is an important functional marker of normal and malignant stem/progenitor cells (5-9). CortesDericks et al. (10) showed that the flow cytometry-based ALDEFLUOR assay could be used to select ALDH ${ }^{\text {high }}$ and $\mathrm{ALDH}^{\text {low }}$ populations to discriminate cancer stem-like cell populations from non-cancer stem-like cells. Sullivan et al. described an enrichment of cancer stem cells (CSCs) in the ALDH ${ }^{\text {high }}$ population in human NSCLC (11). CSCs, supported by the microenvironment, produce more differentiated, metastatic cancer cells, which are detected by and interact with the immune system. There are three phases to this process: elimination, equilibrium and escape (2). The prevention of tumor cell growth and innate immunity constitute the first line of defense (12). This response activates an adaptive immune response against antigens that are specifically expressed by lysed tumor cells and leads to $\mathrm{T}$ cell-dependent tumor control (13). Multiple clinical studies demonstrated that the adoptive cell transfer (ACT) of autologous tumor-infiltrating lymphocytes (TILs) was remarkably effective in melanoma patients (14). Moreover, NSCLC shares similarities with melanoma in mutational burden and sensitivity to immune checkpoint inhibitors $(14,15)$. The presence of TILs positively affects the outcome of NSCLC patients (16). The aim of the present study is to analyze the relationship between LCSCs isolated from human NSCLC patients and CD3+, CD4+ and CD8+ TILs. The results may contribute to new perspectives in the study of interactions between LCSCs and TILs, prompting diagnostic predictive tools and new therapeutic strategies for NSCLC treatment.

\section{Methods}

This cross-sectional study was carried out in accordance to the STROBE (strengthening the reporting of observational studies in epidemiology) statement (17).

\section{Collection of tumor specimens}

The local Ethics Committee of Modena University Hospital approved the present study, which was performed according to the guidelines of the Helsinki Convention. Patients signed an informed consent, and human lung cancer tissues were obtained from 12 consecutive subjects who underwent major surgical lung resection between April 2018 and
September 2018 at the Division of Thoracic Surgery of the University Hospital of Modena for stage I, II, or IIIA NSCLC $\left[8^{\text {th }}\right.$ tumor, node, metastasis (TNM)] (18). Inclusion criteria were: stage I, II, or IIIA NSCLC $\left(8^{\text {th }}\right.$ TNM), age between 18 and 85 years, R0 resection, availability of formalin-fixed, paraffin-embedded surgery specimen from the primary tumor and availability of fresh surgical specimen for fluorescence-activated cell sorting (FACS) analysis. Exclusion criteria were: incomplete resection, unknown TNM status, synchronous tumors, and previous lung cancer. The collection of tumor tissues was carried out during surgery and was set accordingly with the availability of the pathologists involved for our study. The tumor tissue was excised only from the primary lung nodule. The microscopic features of the cancer cells and immunohistochemistry were used to assess the histological diagnosis.

\section{Dissociation of primary tissues}

Tumor tissues were obtained within 1 to $2 \mathrm{~h}$ after surgical removal, washed in sterile Dulbecco's PBS (L1825-BC; Merck Millipore, Italy) and mechanically minced into small pieces ( 2 to $4 \mathrm{~mm}$ ). Minced samples were digested using a tumor dissociation kit in a disposable gentle MACS $^{\text {TM }}$ C-Tube (Miltenyi Biotec, Italy) according to the manufacturer's instructions. Samples were digested for $60 \mathrm{~min}$ at $37^{\circ} \mathrm{C}$ in a gentle MACS Octo dissociator, filtered through $70-\mu \mathrm{m}$ sterile cell strainers, centrifuged at $300 \times \mathrm{g}$ for $5 \mathrm{~min}$, and resuspended in a DMEM and Ham's F12 media mixture (2:1) (Gibco, USA) containing $50 \mathrm{IU} / \mathrm{mL}$ penicillin-streptomycin and $4 \mathrm{mM}$ glutamine. Viable cells were counted using an optic phase-contrast microscope.

\section{ALDEFLUOR assay}

Single-cell suspensions of primary tumor cells were diluted in ALDEFLUOR assay buffer containing BODIPYaminoacetaldehyde (STEMCELL Technologies, Vancouver, BC, Canada). The assay was performed according to the manufacturer's protocol. Briefly, at least 5,000,000 tumor cells were resuspended in ALDEFLUOR BUFFER $\left(5 \mu \mathrm{L} / 10^{6}\right)$ and stained with ALDEFLUOR substrate. Immediately, $5 \times 10^{5}$ cells were transferred to a control tube containing $5 \mu \mathrm{L}$ of diethylaminobenzaldehyde (DEAB), which is a specific inhibitor of ALDH. Control and test samples were incubated for $45 \mathrm{~min}$ at $37{ }^{\circ} \mathrm{C}$ and protected from light. Cells were centrifuged at $300 \times \mathrm{g}$ for $5 \mathrm{~min}$. 

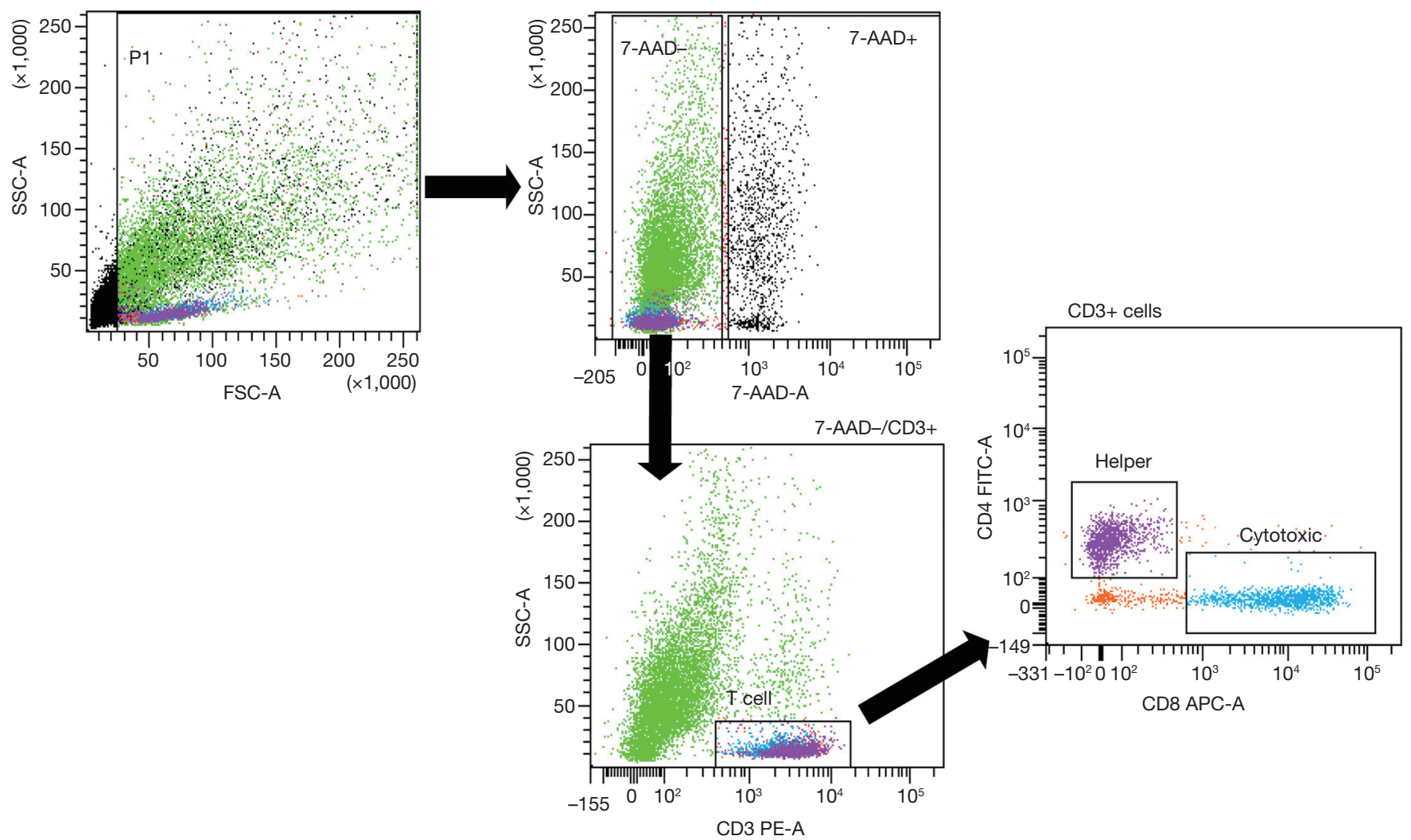

Figure 1 Gating strategy on CD3 + cellular elements and helper or cytotoxic T cells. Lymphocytes were distinguished based on their CD4 or CD8 expression. SSC, side scatter; FSC, forward scatter; 7-AAD, 7-amino-actinomycin D; PE, phycoerythrin; APC, allophycocyanin, FITC, fluorescein isothiocyanate.

The cell pellet was resuspended in $1 \mathrm{~mL}$ of ALDEFLUOR assay buffer. Cell morphology was evaluated using side scatter (SSC) and forward scatter (FSC). Dead cells were excluded using 7-amino-actinomycin D (7-AAD) staining. Cell sorting and ALDH analyses were performed using a FACSAria III (Becton Dickinson, Franklin Lakes, NJ, USA). The results were analyzed using FACSDiva software (Becton Dickinson, Franklin Lakes, NJ, USA). The gating strategy included the $\mathrm{ALDH}^{\text {high }}$ gate, which was set at least one log apart from the ALDH ${ }^{\text {low }}$ gate. Sorted cells were directly lysed for gene expression analyses.

\section{FACS analysis of lymphocytic markers}

Primary tumor cell suspensions were stained with phycoerythrin (PE)-conjugated anti-human CD3 (BioLegend, San Diego, CA, USA), fluorescein isothiocyanate (FITC)-conjugated anti-human CD4 (BioLegend), and allophycocyanin (APC)-conjugated anti- human CD8 (BioLegend). An isotype control sample for each condition was used to exclude the autofluorescence background. Dead cells were excluded using 7-AAD staining. Lymphocytes were gated on CD3 + cellular elements, and helper or cytotoxic $\mathrm{T}$ cells were distinguished based on the sole expression of the CD4 or CD8 marker, respectively (Figure 1). Analyses were performed using a FACSAria III by the same operator, who previously had shared measurement techniques with other operators. Data were analyzed using FACSDiva software.

\section{Immunobistochemistry}

Slides for eight patients were deparaffinized with xylene, rehydrated in a graded alcohol series, and washed in PBS twice for five minutes each. The sections were heated in $10 \mathrm{mM}$ sodium citrate buffer, $\mathrm{pH}=6.0$, for $15 \mathrm{~min}$ in a $95{ }^{\circ} \mathrm{C}$ water bath for antigen retrieval. PBS washes $(5 \mathrm{~min}$ each) were performed until the buffer cooled. Endogenous 
peroxidase activity was blocked via incubation in $3 \% \mathrm{H}_{2} \mathrm{O}_{2}$ at room temperature for $10 \mathrm{~min}$. Blocking serum was added in a dropwise manner at room temperature for $20 \mathrm{~min}$ to reduce the nonspecific background. Samples were incubated with the anti-CD3, CD4, and CD8 antibodies (Ventana Medical System, USA) overnight at $4{ }^{\circ} \mathrm{C}$. Sections were washed in PBS three times for 2 min and incubated with a biotinylated secondary antibody (PK-4001; Vector Labs, USA) for $30 \mathrm{~min}$ at room temperature. The slides were subsequently incubated with ABC-HRP (PK4001; Vector Labs, USA) for $30 \mathrm{~min}$, washed in PBS, and stained with 3,3-diaminobenzidine (DAB). Sections were counterstained with Mayer's hematoxylin, dehydrated, and mounted. Images were collected using a Zeiss Axioskop microscope with a Zeiss AxioCam ICc 3 High-Resolution Microscope Camera. Tissue sections were examined at $10 \times$ magnification to characterize the overall staining pattern and at $20 \times$ magnification for a more accurate evaluation of the cells to assign the appropriate values. Two independent investigators who were blinded to the patients' clinicopathological characteristics performed the scoring of the $\mathrm{CD} 3+, \mathrm{CD} 4+$, and $\mathrm{CD} 8+$ staining according to a previously updated scoring system for colon cancer (19). Sections were scored independently. Immunoreactivity of $\mathrm{CD} 3, \mathrm{CD} 4$, and $\mathrm{CD} 8$ was evaluated using a semiquantitative method based on the positivity of the tumor cells as follows: (I) no or sporadic (approximately $0-5 \%$ ); (II) moderate (approximately 6-25\%); (III) abundant (approximately 26$50 \%$ ); and (IV) highly abundant (approximately $>50 \%$ ) (19).

\section{Statistical analyses}

No formal sample size estimation was carried out in this study, and the number of patients included in the analyses was only based on resource availability. Enrolled patients were those who satisfied the inclusion and exclusion criteria in a time period of 6 months.

Continuous variables were expressed as the means \pm standard deviations and ranges, and categorical variables were expressed as absolute and percentage frequencies. Comparisons between subgroups were performed using the Wilcoxon-Mann-Whitney test for numerical variables.

The outcome variables of interest were (I) ALDH+ expression; (II) CD3 + expression; (III) CD4+ expression; and (IV) CD8+ expression. The measurements of ALDH+ and TILs expression used for the analyses were the percentages of cells calculated based on the total number of 7-AAD-negative cells. The three parameters of interest were the correlations between: (I) ALDH+ and CD3+ expression; (II) $\mathrm{ALDH}+$ and $\mathrm{CD} 4+$ expression; and (III) ALDH+ and CD8+ expression (20). Correlations were assessed using Pearson's linear correlation and Spearman's rank correlation coefficients. We considered two cell populations to be correlated when both linear and rank correlations were observed. The proportion of explained variability for each of the three correlations of interest was also measured by using the R-squared index and is reported as a percentage.

All statistical analyses were carried out in accordance with recently published guidelines (20) and were performed using R 3.4.3 software (The R Foundation for Statistical Computing, Wien, Austria) at the $\mathrm{P}<0.05$ significance level.

\section{Results}

\section{Patients' characteristics}

Surgical specimens were obtained from 12 patients, which were all included in the study. The major clinical and demographic characteristics of the study samples are reported in Table 1. The average age was 71.1 years, $83.3 \%$ of patients were male and all patients were smokers. Clinical stage I was observed in $16.7 \%$ of patients, stage II was observed in $33.3 \%$ of patients, and stage III was observed in $50 \%$ of patients. Nine patients $(75.0 \%)$ had adenocarcinoma, and 3 patients (25.0\%) had squamous cell carcinoma of the lung.

The expression of CD3+, CD4+ and CD8+ cells as determined by FACS is described in Table 1. CD3 expression appeared on $10.8 \%$ of cells (range, $1.0-25.7 \%$ ). CD4 was significantly more expressed than CD8 (5.3\% with range $0.2-18.6 \%$ and $3.2 \%$ with range $0.5-10.9 \%$, respectively). ALDH, CD3, CD4, and CD8 expression was also investigated in subgroups of patients: by gender (males of females), age $(\geq 75,<75)$, histotype (adenocarcinoma, squamous cell carcinoma) and stage (I-II, III) (Table 2). There were no relevant differences in the expression of these markers in the analyzed subgroups (Table 2).

\section{Correlation between ALDH+cells and lymphocyte subsets}

Correlation analyses between ALDH+ and CD3+ cells measured by FACS analysis are reported in Table 3 and Figure 2. We observed a moderate-to-high positive linear correlation (Pearson's correlation $=0.63$, 95\% CI: 0.09 $0.89, \mathrm{P}=0.0270)$ and a moderate-to-high positive rank 
Table 1 Descriptive characteristics of the study patients

\begin{tabular}{lcc}
\hline \multirow{2}{*}{ Features } & \multicolumn{2}{c}{ All patients $(\mathrm{n}=12)$} \\
\cline { 2 - 3 } & Mean $\pm \mathrm{SD} / \mathrm{n}$ & Range/\% \\
\hline Patient characteristics & $71.1 \pm 9.2$ & $52-84$ \\
Age, years & 10 & 83.3 \\
Gender, male & 12 & 100.0 \\
Smoker, yes & 9 & 75.0 \\
Adenocarcinoma & 3 & 25.0 \\
Squamous cell carcinoma & 2 & 16.7 \\
Stage I & 4 & 33.3 \\
Stage II & 6 & 50.0 \\
Stage III & & \\
FACS analysis, \% & & $0.7-12.5$ \\
ALDH+ & $4.6 \pm 3.8$ & $0.2-18.6$ \\
CD3+ & $10.8 \pm 9.1$ & $0.5-10.9$ \\
CD4+ & $5.3 \pm 5.7$ & \\
CD8+ & $3.2 \pm 3.0$ & \\
& &
\end{tabular}

Immunohistochemistry by CD3, CD4, CD8 (15) $(n=8)$

CD3+

No or sporadic

Moderate

12.5

Abundant

6

75.0

CD4+

No or sporadic

2

25.0

Moderate

5

Abundant

1

CD8+

No or sporadic

Moderate

4

50.0

Abundant

4

50.0

SD, standard deviation; FACS, fluorescence-activated cell sorting.

correlation (Spearman's correlation $=0.67, \mathrm{P}=0.0204)$. The proportion of explained variability was $40.1 \%$. Neither linear [Pearson's correlation $=0.34,95 \%$ confidence interval (CI): -0.29 to $0.76, P=0.2802]$ nor rank-based (Spearman's correlation $=0.36, \mathrm{P}=0.2560)$ correlation was found for $\mathrm{ALDH}+$ and CD4+ cells (Figure 3). The proportion of

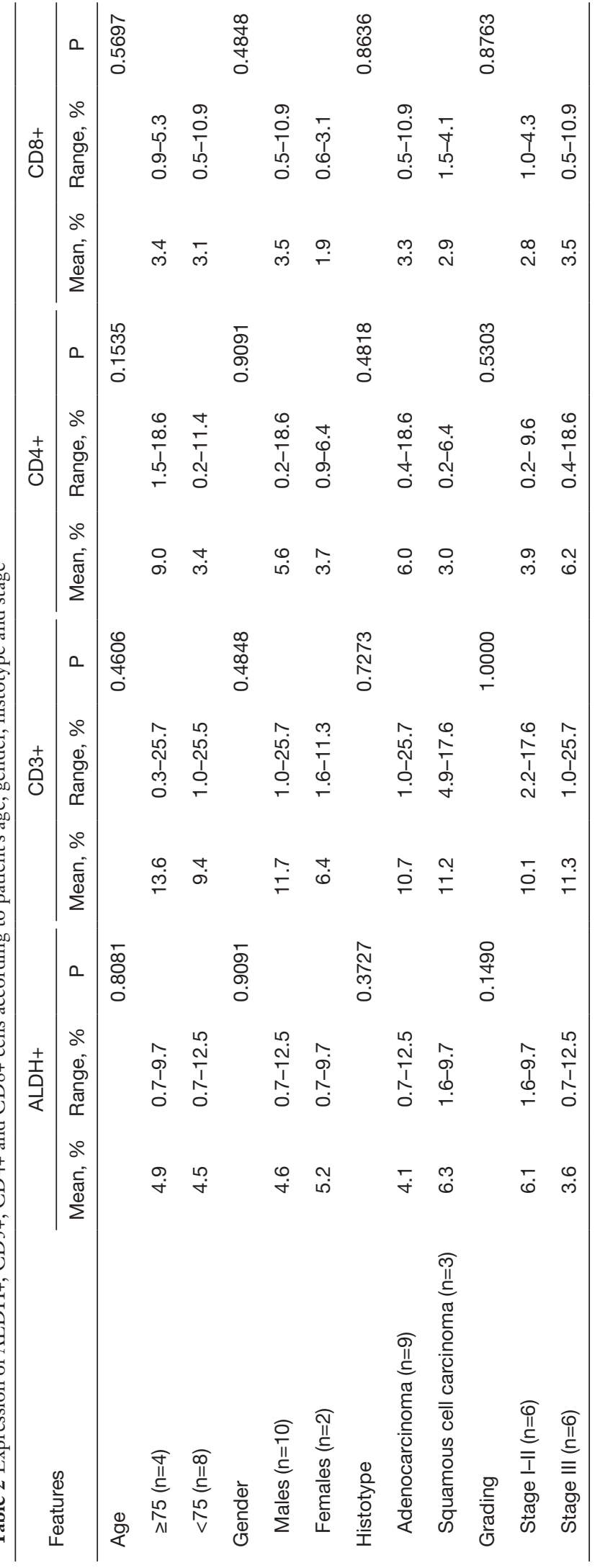


Table 3 Correlation analysis between ALDH+ cells and CD3+, CD4+ and CD8+ cells

\begin{tabular}{|c|c|c|c|c|c|c|}
\hline Antigen & \multicolumn{3}{|c|}{ Pearson linear correlation } & \multicolumn{2}{|c|}{ Spearman rank correlation } & Explained variability (\% \\
\hline CD3+ & 0.63 & $0.09-0.89$ & 0.0270 & 0.67 & 0.0204 & 40.1 \\
\hline CD4+ & 0.34 & -0.29 to 0.76 & 0.2802 & 0.36 & 0.2560 & 11.5 \\
\hline CD8+ & 0.77 & $0.34-0.93$ & 0.0036 & 0.72 & 0.0110 & 58.7 \\
\hline
\end{tabular}

r, correlation coefficient; P, P value; Cl, confidence interval.

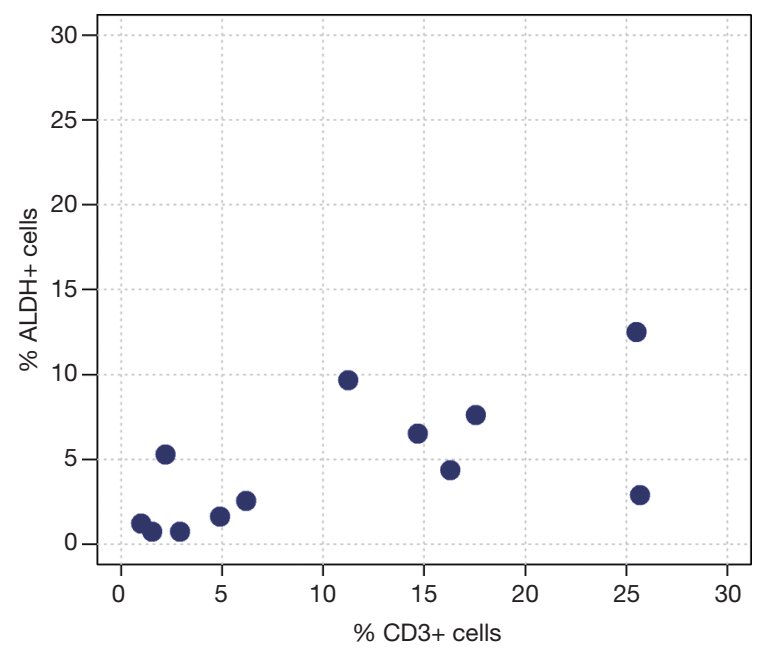

Figure 2 ALDH+ cells compared with CD3+ cells. Scatter plot of $\mathrm{ALDH}+$ cells and CD3+ expression.

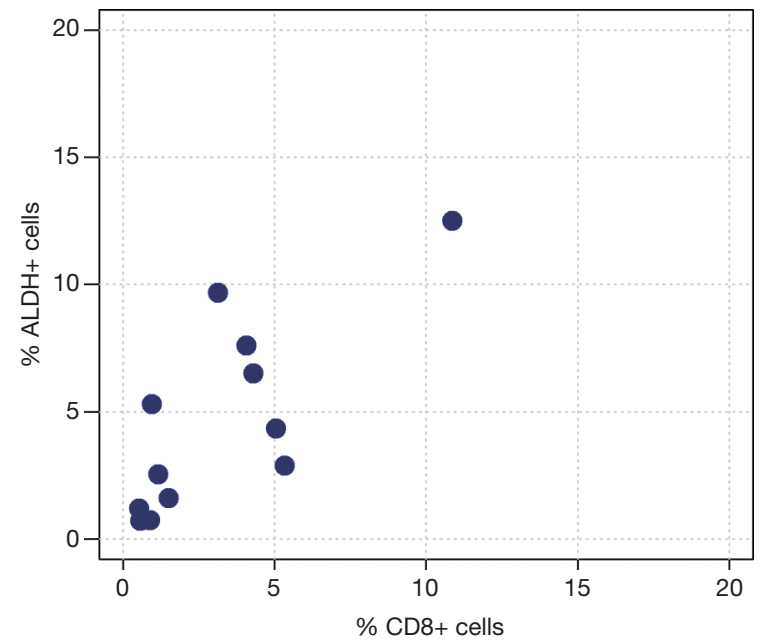

Figure 4 ALDH+ cells compared with CD8+ cells. Scatter plot of $\mathrm{ALDH}+$ cells and CD8+ expression.

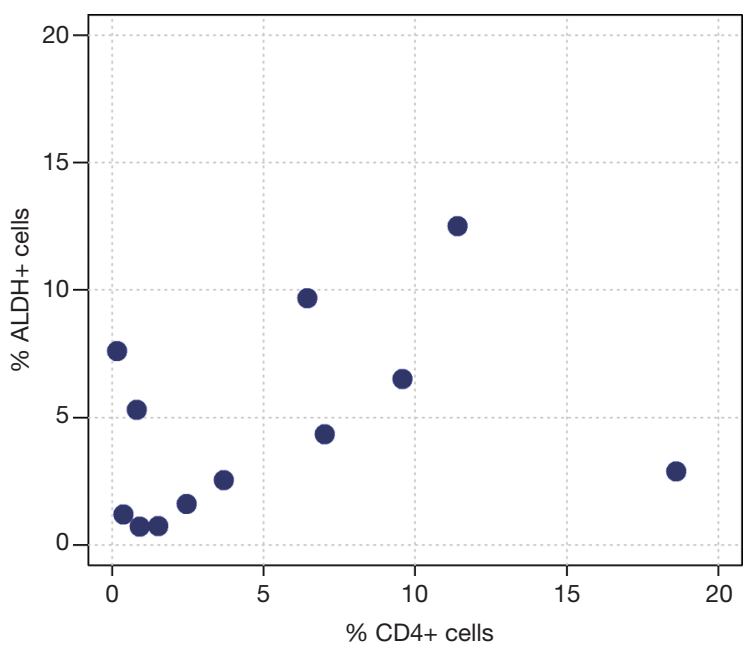

Figure $3 \mathrm{ALDH}+$ cells compared with CD4+ cells. Scatter plot of $\mathrm{ALDH}+$ cells and CD4+ expression.

explained variability was $11.5 \%$. Analyses of the correlations between ALDH+ and CD8+ cells are reported in Table 3 and Figure 4. We observed highly positive linear and rank correlations (Pearson's correlation $=0.77,95 \% \mathrm{CI}$ : $0.34-0.93, \mathrm{P}=0.0036$ and Spearman's correlation $=0.72$, $\mathrm{P}=0.0110)$. The proportion of explained variability was $58.7 \%$. Taken together, all of these data indicate that CD3+ and CD8+ cells were correlated with ALDH+ cells.

\section{Immunobistochemistry}

The expression patterns of $\mathrm{T}$ lymphocyte-associated markers (CD3, CD4, and CD8) were evaluated in NSCLC tumor sections (Figure 5). Cell positivity was scored in 4 classes, as reported (15), and the results are reported in Table 1. The most frequent class for all of the analyzed 


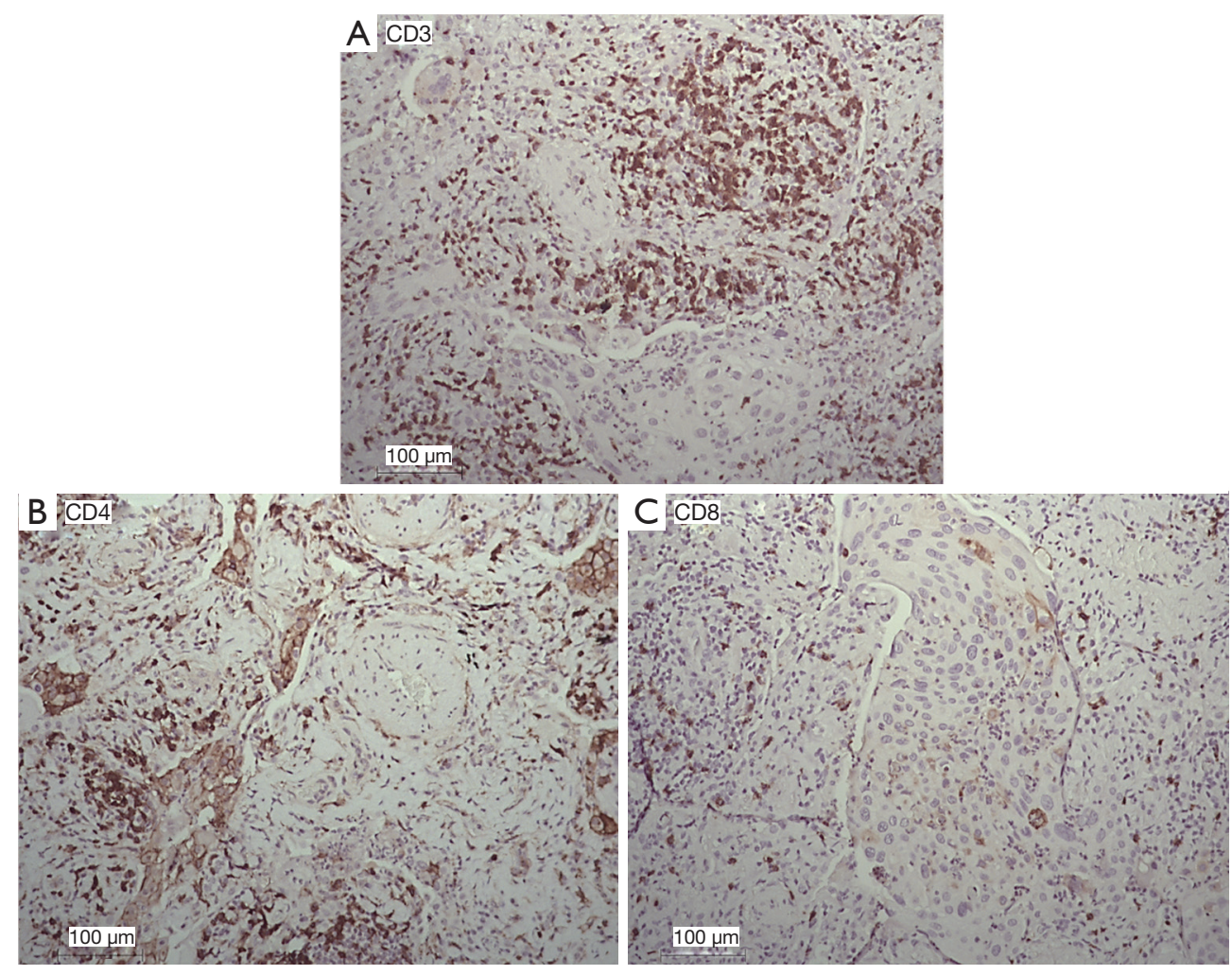

Figure 5 Immunohistochemical analysis and quantification of CD3-, CD4-, and CD8-positive cells in human lung cancer. Paraffinembedded human lung cancer tissues were stained with CD3, CD4 and CD8 antibodies to detect T lymphocytes. (A) Quantification of CD3 + cells in lung cancer; (B) quantification of CD4+ cells in lung cancer; (C) quantification of CD8+ cells in lung cancer. Semiquantitative method based on the positivity of the tumor cells as follows: (I) no or sporadic (approximately 0-25\%); (II) moderate (approximately 2650\%); (III) abundant (approximately 51-75\%); (IV) highly abundant (approximately $>75 \%$ ). Representative images of human lung sections stained with antibodies based on their pathology are shown. Scale bar $=100 \mu \mathrm{m}$.

markers was class 2 (moderate). We observed that the quantification of $\mathrm{CD} 3+$ and $\mathrm{CD} 4+$ also reached class 3 (abundant), and the quantification of CD8+ was greatest in class 2 (moderate) (Table 1), indirectly confirming the data from FACS analyses.

\section{Discussion}

In the present study, we originally observed a relationship between LCSCs and TILs in NSCLC samples obtained from affected patients. First, we found a positive correlation between ALDH+ cells and CD3+ cells and an even stronger correlation between ALDH+ cells and cytotoxic CD8+ lymphocytes. The fact that CD3+ and CD $8+$ expression accounted for $40.1 \%$ and $58.7 \%$, respectively, of the variability of ALDH expression highlights a relevant relationship between these cell populations.
Regarding the role of these lymphocytes, CD3 is the surface marker of mature $\mathrm{T}$ cells and is used to detect both $\mathrm{T}$ helper and $\mathrm{T}$ cytotoxic cells with antitumor activity. Thus, CD3 + cells play a potential dual role in lung cancer via the support of host defense and tumor progression. In contrast, the CD8+ T lymphocyte subpopulation exhibits only cytotoxic activity against cancer cells and may play an important role in antitumor immunity (21). The importance of CD8+ was investigated and clarified in the setting of predictors of clinical outcomes in tumors, including the analysis of their origin, their functional classification, their molecular markers and underlying mechanisms of action against tumors. Clarifications of CD8+ roles could be important to further explorations in the scenarios of prevention, diagnosis and clinical treatment of malignant diseases (21-23). CD8+ effector and T regulatory (Treg) CD8+ cells maintain normal homeostasis in cancer, and 
the ratio $\mathrm{CD} 8+/$ Treg acts as a predictor of clinical outcome in many tumors, such as squamous cell carcinoma of the cervix (24), colorectal cancer (25) and breast cancer (26). The CD8+/Treg ratio was also a potential predictor of response to immunotherapy in mouse models of pancreatic cancer (27), which further supports its potential as an important biomarker and even a driver of the response to immunotherapy. Recent clinical studies are encouraging, showing that immunotherapy for lung cancer alone or in combination with conventional treatments significantly improves patient outcomes (27). However, the exact immune cell composition in NSCLC is not clear, and the scientific community is focusing their attention on specific intratumoral cell compositions that are represented by TILs (24). For the first time to our knowledge, this study raises the attention on the relationship between TILs and a specific lung cancer cell subset. Specifically, LCSCs may also generate unexplored biological insights that may lead to innovative diagnostic strategies for NSCLC in the context of immunotherapy. The reason for this correlation would require deeper investigations and may apparently argue against studies demonstrating an association between high levels of TILs and improved recurrence-free survival in stage 1a NSCLC patients and a reduced likelihood of systemic recurrence (27-29). In addition, a higher frequency of TILs within large node-negative NSCLC correlates with a decreased risk of disease recurrence and improved diseasefree survival (30). Thus, the relationship between high levels of LCSCs, linked with tumor relapse and metastasis (31), and high levels of CD8+ cells could be apparently contradictory.

Indeed, our study suggests an interplay of CD8+ cells and CSCs, indicating that CD8+ T cells could be crucial for cell-mediated antitumor immune response. It has been reported that CSCs express several tumor-associated antigens that could be recognized by CD8+ cytotoxic lymphocytes (32). Possible explanations of the positive correlation detected in our analysis may be that the CSCs stimulate the immune system to initiate a cytotoxic immune response, mediated by CD8+, to suppress these CSCs (32).

However, in our study, we reported no correlation between CSCs and CD4+ cells. We believe that these data reflect what has been previously described in the literature for solid tumors (27-35), defining additional roles for CD4+ T cells, some of which are independent of other lymphocytes, that influence and/or contribute to tumor immunity during carcinogenesis. Consequently, our findings may underline the dualistic role of $\mathrm{CD} 4+\mathrm{T}$ cells in antitumor activity (33). In fact, the absence of correlation between CD4+ T cells and CSCs shown in our data seems to highlight a "mild equilibrium" between these two populations, in which the tumor may have the possibility to grow.

One notable aspect of our study is that CD4+ and CD8+ lymphocytes interplay with LCSCs in the same manner as previously described for epithelial lung cancer cells (29-30).

\section{Limitations}

The main limitations of our study include the low number of patients due to resource availability, the difficulty in obtaining surgical specimens and the small quantity of LCSCs available. In particular, the low sample size could have caused insufficient statistical power; moreover, we were not able to control for potential confounders with a multivariable analysis. However, our research is the first attempt towards showing a correlation between TILs and LCSCs in NSCLC. Further investigations are needed to confirm these data in a larger cohort of patients and to provide more detailed insight on the relationship between TILs and CSCs, such as their joint effect on patient survival. Our research also has the limitation that we did not characterize CD4+ cell subpopulations; however, our results suggest that $\mathrm{CD} 4+$ cells seemed to be involved independently of LCSCs in the immune response against cancer, while CD8+ cells showed a direct dependence on LCSCs.

\section{Conclusions}

In conclusion, in our study, we showed a significant positive correlation between CD8+ T cells and LCSCs, which may prospectively contribute to the development of more immuno-therapeutic approaches for NSCLC patients by targeting CSCs and improving their sensitivity against the cytotoxic activity of CD8+ lymphocytes. Moreover, we strongly believe that even the absence of correlation between CD4+ lymphocytes and CSCs needs to be further investigated. Further, elucidating the mechanisms and interactions among these subpopulations may contribute to the future development of targeted therapies focusing on the different roles of lymphocytes against lung cancer.

\section{Acknowledgments}

The Project has been supported in parts by funds from the Division of Thoracic Surgery of the University Hospital 
of Modena and from the Laboratory of Cellular Therapy of the University of Modena and Reggio Emilia, from unrestricted grant from Myriad Inc. (US) and from the Italian Ministry of Education, University and Research: Departments of Excellence 2017.

\section{Footnote}

Conflicts of Interest: The authors have no conflicts of interest to declare.

Ethical Statement: The authors are accountable for all aspects of the work in ensuring that questions related to the accuracy or integrity of any part of the work are appropriately investigated and resolved. This study, involving human subjects, human material, and human data, has been performed in accordance with the Declaration of Helsinki and has been approved by the Ethics committee at University Hospital of Modena, Modena, Italy, on 17 March 2017 (Prot. N. 914/C.E.). All the patients included in this Study have signed an informed consent before being enrolled.

\section{References}

1. Huang CY, Ju DT, Chang CF, et al. A review on the effects of current chemotherapy drugs and natural agents in treating non-small cell lung cancer. Biomedicine (Taipei) 2017;7:23.

2. Codony-Servat J, Rosell R. Cancer stem cells and immunoresistance: clinical implications and solutions. Transl Lung Cancer Res 2015;4:689-703.

3. Luo J, Zhou X, Yakisich JS. Stemness and plasticity of lung cancer cells: paving the road for better therapy. Onco Targets Ther 2014;7:1129-34.

4. Yakisich JS, Azad N, Venkatadri R, et al. Formation of tumorspheres with increased stemness without external mitogens in a lung cancer model. Stem Cells Int 2016;2016:5603135.

5. Moreb J, Zucali JR, Zhang Y, et al. Role of aldehyde dehydrogenase in the protection of hematopoietic progenitor cells from 4-hydroperoxycyclophosphamide by interleukin 1 beta and tumor necrosis factor. Cancer Res 1992;52:1770-4.

6. Ginestier C, Hur MH, Charafe-Jauffret E, et al. ALDH1 is a marker of normal and malignant human mammary stem cells and a predictor of poor clinical outcome. Cell Stem Cell 2007;1:555-67.
7. Levi BP, Yilmaz OH, Duester G, et al. Aldehyde dehydrogenase $1 \mathrm{a} 1$ is dispensable for stem cell function in the mouse hematopoietic and nervous systems. Blood 2009;113:1670-80.

8. Gentry T, Foster S, Winstead L, et al. Simultaneous isolation of human BM hematopoietic, endothelial and mesenchymal progenitor cells by flow sorting based on aldehyde dehydrogenase activity: implications for cell therapy. Cytotherapy 2007;9:259-74.

9. Traore M, Zhai L, Chen M, et al. Cytotoxic kurubasch aldehyde from Trichilia emetica. Nat Prod Res 2007;21:13-7.

10. Cortes-Dericks L, Froment L, Boesch R, et al. Cisplatinresistant cells in malignant pleural mesothelioma cell lines show ALDHhighCD44+ phenotype and sphere-forming capacity. BMC Cancer 2014;14:304.

11. Sullivan JP, Spinola M, Dodge M, et al. Aldehyde dehydrogenase activity selects for lung adenocarcinoma stem cells dependent on notch signaling. Cancer Res 2010;70:9937-48.

12. Paul S, Lal G. The molecular mechanism of natural killer cells function and its importance in cancer immunotherapy. Front Immunol 2017;8:1124.

13. Chan CJ, Smyth MJ, Martinet L. Molecular mechanisms of natural killer cell activation in response to cellular stress. Cell Death Differ 2014;21:5-14.

14. Reuschenbach M, von Knebel Doeberitz M, Wentzensen $\mathrm{N}$. A systematic review of humoral immune responses against tumor antigens. Cancer Immunol Immunother 2009;58:1535-44.

15. Prelaj A, Tay R, Ferrara R, et al. Predictive biomarkers of response for immune checkpoint inhibitors in non-smallcell lung cancer. Eur J Cancer 2019;106:144-59.

16. Hendry S, Salgado R, Gevaert T, et al. Assessing tumorinfiltrating lymphocytes in solid tumors: a practical review for pathologists and proposal for a standardized method from the international immuno-oncology biomarkers working group: part 2: TILs in melanoma, gastrointestinal tract carcinomas, non-small cell lung carcinoma and mesothelioma, endometrial and ovarian carcinomas, squamous cell carcinoma of the head and neck, genitourinary carcinomas, and primary brain tumors. Adv Anat Pathol 2017;24:311-35.

17. von Elm E, Altman DG, Egger M, et al. The Strengthening the Reporting of Observational Studies in Epidemiology (STROBE) statement: guidelines for reporting observational studies. J Clin Epidemiol 2008;61:344-9. 
18. Detterbeck FC, Chansky K, Groome P, et al. The IASLC lung cancer staging project: methodology and validation used in the development of proposals for revision of the stage classification of NSCLC in the forthcoming (eighth) edition of the TNM classification of lung cancer. J Thorac Oncol 2016;11:1433-46.

19. Dahlin AM, Henriksson ML, Van Guelpen B, et al. Colorectal cancer prognosis depends on T-cell infiltration and molecular characteristics of the tumor. Mod Pathol 2011;24:671-82.

20. Hickey GL, Dunning J, Seifert B, et al. Statistical and data reporting guidelines for the European Journal of CardioThoracic Surgery and the Interactive CardioVascular and Thoracic Surgery. Eur J Cardiothorac Surg 2015;48:180-93.

21. Tian C, Lu S, Fan Q, et al. Prognostic significance of tumor-infiltrating CD8+ or CD3+ T lymphocytes and interleukin-2 expression in radically resected non-small cell lung cancer. Chin Med J (Engl) 2015;128:105-10.

22. Yu Y, Ma X, Gong R, et al. Recent advances in CD8+ regulatory T cell research. Oncol Lett 2018;15:8187-94.

23. Huff WX, Kwon JH, Henriquez M, et al. The evolving role of CD8+CD28- immunosenescent T Cells in cancer immunology. Int J Mol Sci 2019. doi: 10.3390/ ijms20112810.

24. Stankovic B, Bjørhovde HAK, Skarshaug R, et al. Immune cell composition in human non-small cell lung cancer. Front Immunol 2019;9:3101.

25. Lemjabbar-Alaoui H, Hassan OU, Yang YW, et al. Lung cancer: Biology and treatment options. Biochim Biophys Acta 2015;1856:189-210.

26. Zappa C, Mousa SA. Non-small cell lung cancer: current treatment and future advances. Transl Lung Cancer Res

Cite this article as: Masciale V, Grisendi G, Banchelli F, D'Amico R, Maiorana A, Sighinolfi P, Pinelli M, Lovati E, Stefani A, Morandi U, Dominici M, Aramini B. Correlating tumor-infiltrating lymphocytes and lung cancer stem cells: a cross-sectional study. Ann Transl Med 2019;7(22):619. doi: 10.21037/atm.2019.11.27
2016;5:288-300.

27. Kanwal B, Biswas S, Seminara RS, et al. Immunotherapy in advanced non-small cell lung cancer patients: ushering chemotherapy through the checkpoint inhibitors? Cureus 2018;10:e3254.

28. Brahmer JR, Govindan R, Anders RA, et al. The Society for Immunotherapy of Cancer consensus statement on immunotherapy for the treatment of non-small cell lung cancer (NSCLC). J Immunother Cancer 2018;6:75.

29. Yan X, Jiao SC, Zhang GQ, et al. Tumor-associated immune factors are associated with recurrence and metastasis in non-small cell lung cancer. Cancer Gene Ther 2017;24:57-63.

30. Feng W, Li Y, Shen L, et al. Prognostic value of tumorinfiltrating lymphocytes for patients with completely resected stage IIIA(N2) non-small cell lung cancer. Oncotarget 2016;7:7227-40.

31. Moharil RB, Dive A, Khandekar S, et al. Cancer stem cells: An insight. J Oral Maxillofac Pathol 2017;21:463.

32. Huang Z, Yu H, Zhang J, et al. Correlation of cancer stem cell markers and immune cell markers in resected nonsmall cell lung cancer. J Cancer 2017;8:3190-7.

33. Zheng X, Hu Y, Yao C. The paradoxical role of tumorinfiltrating immune cells in lung cancer. Intractable Rare Dis Res 2017;6:234-41.

34. Pan Q, Li Q, Liu S, et al. Concise review: targeting cancer stem cells using immunologic approaches. Stem Cells 2015;33:2085-92.

35. Matsuzaki J, Tsuji T, Luescher IF, et al. Direct tumor recognition by a human CD4(+) T-cell subset potently mediates tumor growth inhibition and orchestrates antitumor immune responses. Sci Rep 2015;5:14896. 